\title{
Video-conferencing in e-commerce website: Effect on perceived service quality and trust
}

\author{
Suleman Shahid ${ }^{1}$, Abdullah Al Mahmud ${ }^{2}$, Omar Mubin $^{3}$ \\ ${ }^{1}$ Tilburg center for Cognition and Communication, \\ Tilburg University, The Netherlands \\ ${ }^{2}$ Swinburne University of Technology, Australia \\ ${ }^{3}$ University of Western Sydney, Australia \\ s.shahid@uvt.nl
}

\begin{abstract}
This study investigates the effect of the presence of live video support in an e-commerce environment on online trust, perceived customer friendliness and perceived quality of service. Participants were asked to rent a car at a car rental website. They needed to find specific information, which was offered, in the form of live video, pre-recorded video or text. Results showed that presence of live video increases perceived support in finding information, perceived customer friendliness and perceived quality of service.
\end{abstract}

Keywords: e-commerce, live video, service quality

\section{Introduction}

E-commerce has shown rapid growth numbers in the last decade. The market for ecommerce goods has doubled in the period between 2005 and 2010 and is still growing with 15 percent [1]. However, there are several aspects of e-commerce, which might withhold consumers from taking part in it. One of the most important disadvantages in e-commerce is based on the lack of personal contact between a customer and a vendor. Salespeople play a key role in the formation of long-term buyer-seller relationships [2]. As the primary link between the buying and selling firms, they have considerable influence on the buyer's perceptions of the seller's reliability and the value of the seller's services and consequently the buyer's interest in continuing the relationship [2]. Personal interaction between customers and vendors does not only affect trust in a company, it also affects perceived service quality by the customer. There are many definitions of the construct 'service quality', but in all definitions personal interaction is a key influencer of service quality [3]. Personal interaction does not only affect online trust. It is also seen as one of the five basic dimensions of service quality [3]. [4] states that customer perceptions of website quality and service quality predict the purchase likelihood for customers. What if there would be more personal contact between the customer and the vendor in e- 
commerce? Would it enhance the trustworthiness of the e-business and the service quality perceived by the customer? This is what we investigate in this study.

The media-richness theory states [6] that a medium can be ranked by its richness, which is based on the possibility to provide direct feedback, the number of cues it can handle, and the opportunity to communicate emotions. [6] also mentioned video conferencing as the richest medium after face-to-face contact. The use of live video streams in e-commerce is an underexposed topic in research. Now that live video is a viable option, there is a need for more research on how it can be implemented in ecommerce environments to make it more personal and how would it affect online trust and service quality.

\section{Method}

The study included a total of 67 participants. Their average age was 27.44 (SD = 6.51) years and all participants were at least 18 years old. The sample was about equally divided between males and females (49\%percent male). The study used a between subject design. There was one independent variable with three levels. The independent variable was the form in which information was presented (live video, pre- recorded video, text). The dependent variables were perceived trustworthiness and perceived service quality measured by the rating on statements, which could be answered on a 5-point Likert-scale.

A website of a fictitious car rental company was created which was a fully working car rental application which participants can use to book a car, find information about cars, insurance, and information about the company. In the 'live video' condition, participants had a possibility to establish a live video stream between themselves and an employee of the company, played by an assistant of the researcher. The participants had an option to switch on or off their webcam in this condition. In the 'recorded video' condition, videos were made in which the same assistant of the researcher provided information on rental conditions, how to use the website and insurance fees. These videos were embedded on the TempCar website. In the 'text' condition, the same information was given using the text format. A questionnaire with statements on trustworthiness and service quality of TempCar was made. The statements on service quality were taken from the SERVQUAL questionnaire, which were adjusted for websites by [5]. The statements represent different aspects of trust and service quality in e-commerce environments and could be answered on a 5-point Likert-scale with answers varying from 1 being 'Very poor' to 5 'Very good'.

Participants were instructed to find information on renting a car website. The car needed to meet specific requirements. They had to find information on insurance fees, renting costs and whether or not their friend was allowed to drive in the car. In the live video condition, participants could not find the answer to all the questions on the website, but were steered into asking for help in a live video chat. For instance, when they were looking for information about insurance fees, they would find a page with information on insurance which said they could use the live help function on the website for information on insurance fees (see figure 1). In the pre-recorded video condition, participants could only find the answer in the videos displayed on the 
helpdesk page. In the 'text condition', the information was presented in a textual form on a FAQ page. For the text page to be as similar as possible, the required information can be found in the middle of a paragraph, instead of the usual 'question-answer' form used in FAQ pages. This mimics the characteristics of video, where you cannot just scan the video to find the information you are looking for. Participants have to watch the video as a whole to find the information.

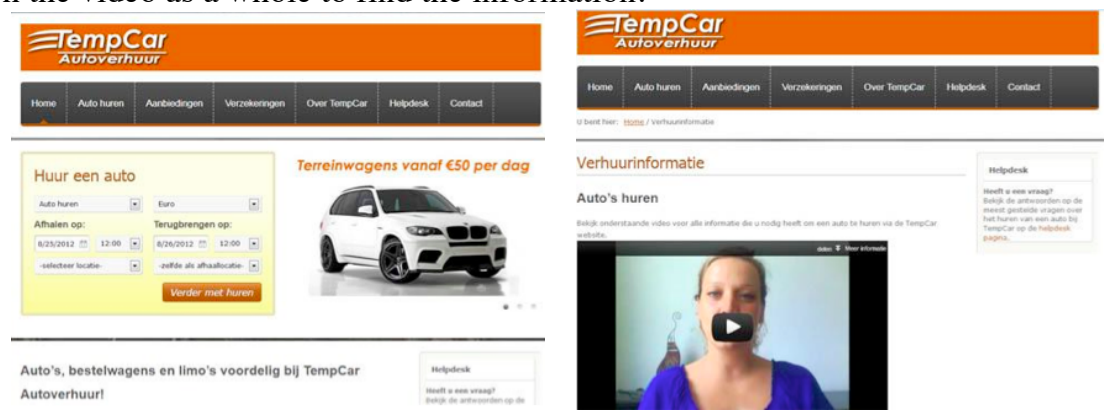

Figure 1: TempCar website and videos

\section{Results}

A one-way ANOVA was run to find differences in the mean scores on statements on perceived trust and service quality between the three conditions (live video, prerecorded video, text). All mean scores can be found in Table 1. In all conditions only those participants who used these feature to get actual help were included. When significant differences were found, Gabriel's test was used as a post-hoc analysis, because the sample sizes were unequal (4 more participants in the live condition as compared to other two). Gabriel's test can be unreliable when the assumption of homogeneity has been violated. This was tested for by Levene's test and a violation of homogeneity was not the case in the sample used for this study.

Table 1: Mean scores of participants in three conditions

\begin{tabular}{llll}
\hline & Text & Video & Live video \\
\hline Perceived support & $3.67(.66)$ & $3.33(.80)$ & $4.20(1.04)$ \\
Customer friendliness & $3.48(.68)$ & $3.62(.59)$ & $4.56(.77)$ \\
Quality of service & $2.57(.60)$ & $3.43(.75)$ & $4.04(.79)$ \\
Trustworthiness & $3.62(.81)$ & $3.48(.87)$ & $3.63(.84)$ \\
\hline
\end{tabular}

Significant differences were found between the scores on perceived support in finding the required information in the three conditions. A one-way ANOVA showed a strong effect of the medium used to present the information perceived support, $\mathrm{F}(2,64)=5.98, \mathrm{p}=<.01, \omega=.35$. A live video increases the perceived support by a company. An even greater effect was found for perceived customer friendliness, $\mathrm{F}(2,64)=17.16, \mathrm{p}=<.001, \omega=.57$. The perceived customer friendliness was indeed higher in the live video condition. The scores on perceived quality of service were 
significantly higher among users of the live video help than among the other participants, $\mathrm{F}(2,64)=4.61, \mathrm{p}=<.05, \omega=.31$. A one-way ANOVA for the trustworthiness dimension showed no significant differences between the three conditions. The post-hoc analysis shows that participants in the live video condition rate the perceived support, quality of service and customer friendliness significantly higher than the other two conditions but there was no difference between the text and record video conditions.

\section{Discussion and Conclusion}

This study investigated how the presence of a live video effects perceive support customer friendliness, quality of service and trustworthiness of an e-commerce website. The results clearly show that use of live video has a positive effect on almost all dimensions. Despite being instructed repeatedly to contact the live video helpdesk to find all the required information, 37 percent of the participants in the live video condition did not contact the live video help. There seemed to be resistance to contact a company to find information on the Internet, even though participants could not find all the required information needed to complete the task. Although participant were not forced to switch on their webcam and company representative's video stream was enough to get the information, participants stated they were resistant to be seen by somebody, while being in front of a computer. There seemed to be serious privacy issues among the participants when it comes to being seen via a webcam connection. This is a strong indication that all the steps in using live video should be explained very clearly to reduce any uncertainties before users press a button to call for live video help. Interestingly, most participants who did use the live video help rated the experience as very good $(M=4.48, S D=92)$. In future research we would like to test live text chat, live audio chat and live video chat to find out which form of live contact scores best on online trust, customer friendliness etc.

\section{References}

1. Internetkassa (2011). Online verkopen trekt weer aan. Retrieved on March 27, 2015, from http://www.internetkassa.nu/nieuws/738-online-verkopen-trekt-weer-aan.html.

2. Biong, H. and Selnes, F. The Strategic Role of the Salesperson in Established Buyer-Seller Relationships. Working Paper Report No. 96-118, Marketing Science Institute, Cambridge, MA (1996)

3. Dabholkar, P.A., Thorpe, D.I. \& Rentz, JO. A Measure of Service Quality for Retail Stores: Scale Development and Validation. Journal of the Academy of Marketing Science, 24(1), (1996) 3-16

4. Cao, M., Zhang, Q.Y. and Seydel, J., B2C e-commerce web site quality: anempirical examination, Industrial Management \& Data Systems, Vol. 105 No. 5, (2005) 645- 61

5. Cox, J. and Dale, B.G. Service quality and e-commerce: an exploratory analysis, Managing Service Quality, Vol. 11 No. 2, (2001) 121-31

6. Daft, R., \& Lengel, R. Organizational information requirements, media richness and structural design. Management Science, 32: 554-571 (1986) 